\title{
Trust Recipes for Enhancing the Intention to Adopt Conversational Agents for Disease Diagnosis: An fsQCA Approach
}

\author{
Eduard Anton \\ Osnabrück University \\ eduard.anton@uos.de
}

\author{
Thuy Duong Oesterreich \\ Osnabrück University \\ toesterreich@uos.de
}

\author{
Christian Fitte \\ Osnabrück University \\ christian.fitte@uos.de
}

\author{
Frank Teuteberg \\ Osnabrück University \\ frank.teuteberg@uos.de
}

\begin{abstract}
In this study, we examine the configurations of trust-enhancing factors that determine the intention to adopt conversational agents (CAs) for disease diagnosis. After identifying trust factors influencing the behavioral intent to adopt CAs based on the information systems acceptance research field, we assigned 201 participants to use the mobile Ada application and surveyed them about their experience. Ada is a medical diagnostic CA that combines patients' symptoms with their medical history and provides diagnostic suggestions. The collected data was analyzed using a fuzzy set qualitative comparative analysis to capture the causal complexity of trust. We identified several configurations of trust-enhancing factors affecting the intention to adopt the $C A$. In particular, our results show that the adoption intentions are strongly determined by trust factors associated with the performance dimension. Furthermore, we derived two propositions for the development of CAs for healthcare purposes and elaborated implications for research and practice.
\end{abstract}

\section{Introduction}

Traditionally, medical diagnostic services require interpersonal interactions [1] and are characterized by high information asymmetries [2]. While patients consult doctors in the expectation that medical disorders are relieved or cured, it is doctors who, as the center of medical knowledge, make diagnoses and prescribe appropriate therapies. Since patients are usually unable to assess the accuracy of diagnoses and effectiveness of treatments, trust in the attending physician is required [3]. However, trust in physicians and the healthcare sector has recently declined internationally because of misdiagnosis, medical errors or overtreatment due to economic reasons [4].

With the rise of artificial intelligence (AI) in healthcare, conversational agents (CAs) can provide detailed information from independent sources, reducing information asymmetries and empowering patients to verify doctors' recommendations [5]. CAs are computer-based assistants which are able to interact with users in natural language [6]. Applied in medical diagnostics, the systems can utilize AI by analyzing vast amounts of different types of health data to provide individualized and contextual advice for patients [7]. CAs for disease diagnosis, such as Ada Health [8], are able to ask follow-up questions to narrow down symptoms and specify medical diagnoses [5]. This allows patients to self-diagnose without consulting a doctor, which can avoid long waiting times for a medical appointment or provide a cost-effective alternative, especially in countries where medical services are expensive [9]. The output of such CAs can be interpreted as complementary suggestions beyond medical diagnostics. However, since these medical recommendations generally cannot substitute for or be validated by a physician, the users' confidence in them ultimately depends on the trust in the reliability of the underlying system and information [5]. The aim of this study is to address the factors that influence trust affecting the adoption of CAs like Ada Health for medical diagnosis.

Previous research already highlighted the importance of trust for adopting CAs for disease diagnosis $[5,10,11,12]$ or other AI-based diagnostic systems [4] and medical assistance devices [13, 14]. However, the perspective on trust in these studies does not address the complexity of this relational phenomenon, especially in scenarios involving aspects of automation, which is difficult to explain by the effect of individual constructs $[15,16]$. The distinctive feature of CAs is their ability to mimic humanlike nature through their natural conversational flow, which can encourage users to "treat computerized agents as social actors, and form social relationships that involve trust" [17:75]. Thus, trust in CAs represents a causal framework of different sociotechnical nuances of several dimensions [18]. Regression analyses often reach their limits when, especially in complex scenarios, multiple interaction effects must be considered in a single analysis. Then, nuances are usually missed as 
variance in the observation of effects [19]. To cope with this complexity, we propose an alternative approach to previous quantitative methods $[4,10,14]$ by leveraging configurational theory to examine the effects of trustenhancing factors on the adoption of CAs. Therefore, we pose the following research question (RQ): What configurations of trust-enhancing factors determine the intention to adopt AI-based conversational agents for disease diagnosis?

In order to answer the RQ, we conducted an online survey with 201 participants and analyzed the data using a fuzzy set qualitative comparative analysis (fsQCA), a configurational approach comprising fuzzy set logic with Boolean algebra to examine causal pathways for explaining an outcome $[20,21]$. Our results contribute to a better understanding of the complex trust-building process towards CAs for disease diagnosis by providing transparency into the inherent complex process that can follow different causal pathways.

\section{Theoretical background}

\subsection{Conversational agents for disease diagnosis}

With increasing processing power and advances in natural language processing (NLP), conversational agents have become widespread as text- or speech-based interfaces in domain-specific or general purpose domains. They can capture user intent and provide static or dynamic responses based on rule-based or selflearning capabilities [22]. CAs for disease diagnosis represent a domain-specific application and allow patients to self-diagnose by reporting symptoms and personal data to a CA engine that lead the user in one or several steps to a diagnosis [12]. The benefit is that it reduces the inhibition threshold for asking uncomfortable questions, facilitate the access to healthcare information and relieve physicians by reducing unnecessary appointments [23]. Self-learning CA engines utilize AI-related techniques such as machine learning algorithms and NLP to learn from structured and unstructured medical data, to draw conclusions from data patterns in order to calculate disease probabilities and to adjust the response paths to specific symptoms [24]. However, the use of diagnostic systems in healthcare is still considered risky, as the non-detection of illnesses or misinterpretations of symptoms can have dramatic consequences [4]. Therefore, current legal regulations in most countries of the Western Hemisphere do not allow medical diagnostics solely based on automated decisions [5]. Automated decisions are defined as "decisions by technological means without human involvement" [25]. To date, a doctor or medical professional must be involved to evaluate and contextualize algorithmic results [26]. While this is ensured for decision support systems developed to assist physicians (e.g., WFO) [26]), it is not for CAs providing diagnostic suggestions to patients as end-users. Therefore, users of such CAs are exposed to performance risks that can lead to distrust [12] and the rejection of such tools [9]. Trust in the CA and the underlying information is essential to reap the benefits for the individual and the healthcare system. CA research often focuses on anthropomorphic design features to mimic human-like aspects to increase trustworthiness [27] or examines trust in the context of technology acceptance models $[11,12]$. However, there is a lack of configurational approaches that account for the causal complexity of trust in CAs.

\subsection{The causal complexity of trust in information systems research}

Trust is a social construct empowering individuals with a relational evaluation instrument to assess uncertainty and risk exposure in almost every type of interaction and transaction [28]. The perception of trust is subjective and its formation depends on the contextual condition, the nature of expectations and the object of dependence [29]. In addition, the construct includes a dynamic component which is reflected in the distinction between initial and continuous trust [30]. These contextual-dependent, dynamic and subjective components highlight the causal complexity of trust [29].

Trust as an uni- or multidimensional construct is frequently studied in the information systems (IS) acceptance research. In this literature stream, the nature of trust is transferred from interpersonal interactions to study its effects on the adoption of technological artefacts [17, 29, 31]. The nomological validity of trust is commonly examined by theoretical frameworks such as the technology acceptance model (TAM) [17, 32] or the unified theory of acceptance and use of technology (UTAUT) [4]. In healthcare, such models are employed to explain the acceptance of AI-based artefacts such as CAs [10], medical assistance devices [13] or opthamalic AI devices [14] from different perspectives such as those of healthcare professionals [4] or patients [5].

Research utilizing such structural models demonstrated the significance of trust by measuring its direct or indirect effect on the behavioral intention (BI) to use the artefact under investigation and emphasized the effects of antecedents on trust as a dependent variable $[4,5,14]$. IS acceptance research in healthcare generally examines the effects using variance-based structural equation modelling (SEM) to assess relationships between the measured variables and latent constructs $[4,14]$, or it focuses on qualitative methodologies $[5,13]$. Previous quantitative approaches 
assume symmetrical relationships between trust and other variables to explain the resulting net effects, as is the case with initial trust as an independent variable that has a positive effect on the dependent variable behavioral intent (BI) [4]. A symmetric relationship between those two variables suggests that the intention to adopt a particular artefact increases or decreases with the level of trust. This implies that the occurrence of the outcome presupposes the existence of a relationship between initial trust and BI (necessary and sufficient condition) [20]. However, since trust is fragile, it can easily be destroyed by unfulfilled subjective expectations. The causal complexity for gaining or losing trust rather suggests an asymmetric relationship that captures subjectivity and nuances of the social construct [13]. As we argue that different causal conditions can result in the same outcome, we follow a configurational approach to examine the holistic effects of trust-enhancing variables leading to intentions to adopt CAs for disease diagnosis by utilizing fsQCA [33]. To emphasize this approach within the context of the paper, we understand trust as the "willingness of users to provide confidential information, accept the recommendations, and follow the suggestions" [34:1].

\section{Research model}

With the configurational tenet of this study, we consider the holistic effect of conditions affecting an outcome [35]. Hengstler et al. [13] revealed that trust in applied AI is built on conditions of three bases: purpose, process and performance. Purpose refers to the faith in the trustee's intentions to fulfil all obligations relating to the setting of an artefact $[13,15]$. Thereby, the motives of the provider responsible for the artefact must be included [36]. The trust base process is nurtured by the comprehensibility and auditability of the used AI artefact and the supported process [13]. Performance represents the competence or ability of an artefact to achieve the user's targeted goal [15]. The three dimensions are indicators for measuring trust in IS [16]. Our research model considers the formation of initial trust based on the mentioned trust bases. Initial trust is built after a first trial of an artefact [16]. It represents a subjective, relational tool to evaluate the intention to use a technology in the future. Thus, it is a construct for measuring the state before individuals intend to adopt a new technology [31]. Fan et al. [4] demonstrated that, from the perspective of healthcare professionals, initial trust has a positive influence on BI to use AI-based diagnostic systems. As in the study of Fan et al. [4], our research participants had no prior experience with AIbased diagnostics and had to assess their trust in the technology before deciding to adopt it. Hence, we include the initial stage of trust to our research model by considering the dynamic components of the trust building and adoption process [16, 30].

In order to answer our RQ, we utilize the three trust bases for applied AI and operationalize them with constructs from technology acceptance research. Thus, we contemplate initial trust as a configuration of various trust building constructs contributing to the respective trust base. As shown in Figure 1, we assigned the constructs to their respective dimensions by considering their trust building capacity reflecting the trustors' perceptions of the trustees' motives (purpose), the ability to achieve the trustors' goals (performance) and the comprehensibility during usage (process) [15].

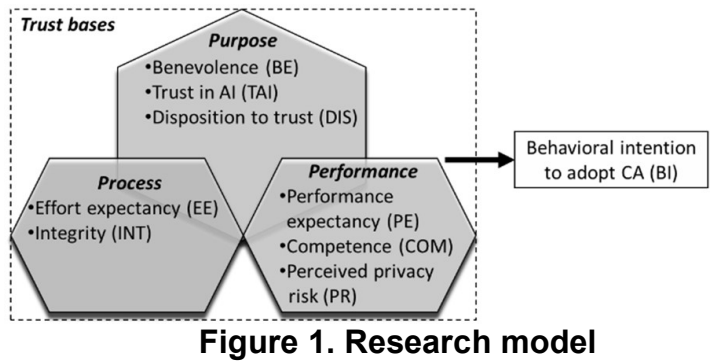

In conventional medical settings, trust is generally determined by patients' expectations with regard to the competence and the benevolence of the trustee [37]. Confidence in physicians is normally rooted in their license to practice and in the socially respected medical profession that adheres to integrity and values that puts the well-being of patients first. Thus, patient confidence in the abilities and the intentions of physicians is strengthened by previous experience with medical professionals and institutional characteristics [38]. The adoption of CAs for disease diagnosis requires a paradigm shift, as trust has to be placed in automated decisions [5]. The trustworthiness of AI-based artefacts is yet controversially discussed $[30,39]$. While some authors consider it wrong to personalize AI, as a rather "faulty surgical instrument" [39] that cannot be held responsible and to which no morality can be ascribed, others are convinced that the human-like presentation of AI artefacts is essential to create trustworthiness and acceptance [30]. Regardless of how much agency is assigned to AI, a CA for disease diagnosis remains a technological artefact developed and operated by a provider. Prior acceptance research distinguished between trust in a provider and a technological agent by empirically demonstrating that trust in the provider can have a positive effect on trust in a technology and the intention to adopt it $[5,16,31]$. Trust in the provider is an institution-based form of trust incorporating the outcome of structural assurance and situational normality; hence, it comprises components similar to those important for building trust in a physician. Trust 
in the provider is operationalized by users' perception of the benevolence, the competence and the integrity of the provider [16].

Purpose. To account for the "faith in intentions" [13:106] that characterizes the purpose dimension, we have included benevolence (BE) as part of institutionbased trust in this dimension. In addition, we consider aspects of structural assurance and situational normality addressing the context trustors are embedded in. The underlying idea is that trustors who have a positive perception about the present user scenario and the structural safeguards can build trust more easily [16, 31]. Söllner et al. [16] found empirical evidence that multiple targets of trust relationships matter to understand the intentions for adopting a particular technology. Thus, the authors incorporated aspects of structural assurance and situational adoption in the construct "trust in the internet". We adopt this approach and consider trust in $A I$ (TAI) as a pendant in our context. We examine this construct with benevolence, integrity and competence separately for providing more explanatory power with regard to institution-based trust in the configurational approach.

Disposition to trust (DIS): Trust as a social construct requires interpersonal ties based on former encounters, with each experience influencing the propensity to trust [32]. If trust in the provider could not be established previously, the disposition arises from the trusting stance and the general faith in humanity. Thus, especially in scenarios of initial trust, disposition to trust can have a direct effect on trusting beliefs as well as on BI [40]. Since our participants had no prior experience with CAs, our setting is suitable to consider the construct in our configurational approach [4].

Process. Effort expectancy (EE): The experience regarding the initial effort required to handle a new system is especially important for the BI to adopt a technology. EE, a construct of the UTAUT, is utilized to measure the ease of use of a system [41, 42]. Studies have shown that both EE $[4,5]$ and its TAM pendant perceived ease of use (PEOU) $[16,17,43]$ are crucial for trust in IS. In the context of CAs, the understanding how to use the application enables users to faster and easier reach their desired goal of obtaining a diagnosis, which can lead to trust in the application [5]. The EE thus addresses the performance of a technology on a process level. Integrity (INT) as part of institution-based trust characterizes the confidence of users in the consistency of the provider's services and their usability [15].

Performance. Performance expectancy (PE): Performance is a technological characteristic that defines the "competence of AI in completing tasks and finishing those tasks in a consistent and reliable manner" [30:51]. PE (or the related perceived usefulness (PU) of the TAM) is a determinant of BI in the original UTAUT [42]. However, Fan et al. [4] show that $\mathrm{PE}$ can act as an antecedent to initial trust in AIbased diagnostic systems. Thus, we include PE as a determinant of initial trust in the performance base. The pendant of PE directed at the vendor is competence (COM) as part of institution-based trust. This construct questions the vendor's ability to assess symptoms and derive diagnoses from them.

Perceived risk (PR) is neither part of the original TAM [44] nor the UTAUT [45]. Nevertheless, risks must be part of the causal trust recipe, since trust involves the willingness to take risks [46]. In the context of AI, privacy risks are of particular concern [5, 47], because technologies such as machine learning require a large amount of personal data to work effectively [30]. The perceived privacy risk can increase concerns about the performance and raise questions about its ability to achieve users' goals [48].

\section{Research methodology}

Our study follows a multi-step research approach consisting of the three phases study design, data collection and data analysis. It is informed by the literature on configurational analysis $[33,49]$ as well as related IS research $[35,50,51]$.

\subsection{Study design and sample}

Study design. The main purpose of this phase is to identify and explore factors that determine the intention to adopt CAs for disease diagnosis. Based on literature from the IS acceptance research field, we first developed a research model that incorporates constructs adopted from various validated measures of previous acceptance research (cf. Section 3). We employed a quantitative method and designed a formal questionnaire to validate the presented constructs. The items of the questionnaire were measured using a seven-point Likert scale, allowing answers from strongly disagree (1) to strongly agree (7). The participants were introduced to Ada and its technology before being requested to test the app prior to answering the questions by trying to find a diagnosis to a hypothetical or actual medical case related to themselves or another person. To ensure that all participants have tested the Ada app, we included a question directly at the beginning of the survey in which the participant has to explicitly confirm that he or she has downloaded and tested the app based on the hypothetical or actual medical case. The results of a pretest study with 11 participants were used to improve the questionnaire design.

Data collection and sample. The empirical data for this study was collected via an online survey among 
undergraduate students of a German university. Although students may represent a unique sample at the first sight, we consider them an appropriate target group in the specific setting of our research for several reasons. As young adults and regular Internet users, students constitute a relevant user group of the ADA app [5, 10]. Since the intended population for our study are potential users of ADA, students can be considered a major part of the population of interest according to recommendations in IS literature [52]. The online survey was incentivized by the opportunity to win a gift voucher. The survey link was sent to 301 students of an IS course and yielded in 218 responses, which corresponds to a response rate of $72.4 \%$ and thus exceeds the recommended threshold of $30 \%$ [53]. Since our primary aim is to examine the impact of trust-related factors on the intention to adopt CAs for disease diagnosis at an initial stage, apart from five incomplete responses, we also removed the twelve responses of those who claimed to already use Ada. In this process, we checked all responses which significantly deviate from the average duration to identify potential manipulating responses. In the subsequent step, the remaining 201 valid responses were statistically examined. A brief overview of the demographic data of the total sample is depicted in Table 1.

Table 1. Descriptive statistics of the respondents $(n=201)$

\begin{tabular}{|c|c|c|c|c|c|}
\hline \multicolumn{2}{|c|}{ Characteristic } & $\%$ & \multicolumn{2}{|c|}{ Characteristic } & $\%$ \\
\hline \multirow{2}{*}{ Gender } & Male & $61 \%$ & \multirow{2}{*}{$\begin{array}{l}\text { Grad- } \\
\text { uate } \\
\text { Level }\end{array}$} & Bachelor & $97 \%$ \\
\hline & Female & $39 \%$ & & Master & $3 \%$ \\
\hline \multirow{4}{*}{ Age } & $18-20$ & $53 \%$ & \multirow{4}{*}{ Major } & IS & $26 \%$ \\
\hline & $21-25$ & $44 \%$ & & Economics & $73 \%$ \\
\hline & $26-30$ & $2 \%$ & & & 10 \\
\hline & $30<$ & $1 \%$ & & & $1 \%$ \\
\hline
\end{tabular}

\subsection{Data analysis}

Measurement and validation. For measuring the utilized constructs, we performed a confirmatory factor analysis (CFA). CFA enabled us to test the fit by which the data represents our pre-specified measurement model [54]. Using the Harman's single-factor test, which considers the variance by the measurement method rather than the actually observable variables, we test for common method bias. A single factor is explaining $25 \%$ of the overall variance in our data which is less than the threshold of $50 \%$, thus indicating no significant impact of common method bias [55]. We estimated internal consistency reliability by calculating Cronbach's alpha $(\alpha)$ and the composite reliability (CR) for the observed variables. The estimates for our constructs exceed the recommended thresholds of 0.7
[54]. In a next step, we checked the convergent validity by calculating the Average Variance Extracted (AVE). The estimates are ranging between 0.529 and 0.870 and are thus all above the cutoff value of 0.5 [54]. To confirm discriminant validity, we considered the Fornell-Larcker criterion which requires the square root of the AVE of each latent construct to be less than the absolute value of the correlations with another factor [56]. In addition, we calculated the heterotrait-monotrait (HTMT) ratio of correlations to confirm that our constructs differ from each other. We achieved values below the upper limit of 0.85 assuring discriminant validity [57]. To enhance comprehensibility and transparency, detailed results of the quantitative approach and analyses are given as supplementary material at https://bit.ly/3mQZaaN.

fuzzy set qualitative comparative analysis. fsQCA is an appropriate method to combine quantitative variables and qualitative case-based practices to investigate complex causal phenomena [58], such as cause-effect relationships of trust building variables affecting users' intention to adopt CAs for disease diagnosis [33]. The underlying fuzzy set logic suggests that the extent to which a certain set can belong to a given condition ranges in the interval between 0 and 1 (degree of membership). We define membership associations to build causal combinations by using operators from set theory to form a causal blueprint for explaining an outcome [49]. The relationships between the outcome and the combination of variables can be necessary or sufficient for the studied outcome [49]. Conducting an fsQCA comprises the steps calibration and analysis of necessary and sufficient conditions.

Calibration is the process of transforming our composite scores of the latent constructs into fuzzy set membership scores between 0 and 1 . For this purpose, it is necessary to set three qualitative anchor points for the survey data known as full membership, crossover point and full non-membership [33]. We adopted the calibration strategies of Duarte and Picoto [59] by setting the anchor values to the $5^{\text {th }}$ percentile for full non-membership, the median for the crossover point, and the $95^{\text {th }}$ percentile for full membership.

The analysis of necessary and sufficient conditions was conducted based on the calibrated data by using the fsQCA 3.0 software. In this context, necessity and sufficiency refer to a set-theoretical analysis of empirical cases in which, necessary conditions are observed as supersets and sufficient conditions as subsets of an outcome. For an independent variable to be considered a superset of an outcome, every empirical instance that has membership in the outcome must also have membership for the independent variable [33]. Since this state is empirically rarely reached for all cases, a consistency threshold of 0.9 is set to qualify an 
independent variable as a necessary condition [60, 61]. An independent variable is a sufficient condition for an outcome if the outcome is a superset of the independent variable. In order to identify sufficient conditions for an outcome, we create a truth table, showing $2^{\mathrm{k}}$ causal combinations ( $k$, for number of variables), that can potentially influence the outcome. To derive the configurational solutions, we used a frequency cutoff of $\geq 3$ empirical observations, considering our sample size, and a consistency cutoff above the recommended threshold of at least 0.75 , at 0.85 [20, 21, 62]. Subsequently, we followed the threshold recommended by Schneider and Wagemann [58] for proportional reduction in inconsistency scores (PRI) higher than 0.65 [63].

\section{Analysis of necessary and sufficient conditions}

The analysis of necessary conditions based on the calibrated data yielded in the estimates depicted in Table 2.

Table 2. Analysis of necessary conditions

\begin{tabular}{|c|c|c|c|c|}
\hline \multirow{2}{*}{ Condition } & \multicolumn{2}{|c|}{ BI as outcome } & \multicolumn{2}{|c|}{$\sim \mathrm{BI}$ as outcome } \\
\hline & Cons. & Cov. & Cons. & Cov. \\
\hline $\mathrm{BE}$ & 0.744 & 0.570 & 0.635 & 0.604 \\
\hline$\sim \mathrm{BE}$ & 0.687 & 0.602 & 0.670 & 0.730 \\
\hline TAI & 0.728 & 0.711 & 0.590 & 0.594 \\
\hline$\sim \mathrm{TAI}$ & 0.585 & 0.580 & 0.714 & 0.730 \\
\hline DIS & 0.680 & 0.675 & 0.616 & 0.630 \\
\hline$\sim$ DIS & 0.626 & 0.613 & 0.682 & 0.687 \\
\hline $\mathrm{EE}$ & 0.746 & 0.680 & 0.620 & 0.584 \\
\hline$\sim \mathrm{EE}$ & 0.544 & 0.581 & 0.660 & 0.728 \\
\hline INT & 0.798 & 0.775 & 0.548 & 0.549 \\
\hline$\sim$ INT & 0.535 & 0.534 & 0.775 & 0.799 \\
\hline $\mathrm{PE}$ & 0.818 & 0.796 & 0.502 & 0.504 \\
\hline$\sim \mathrm{PE}$ & 0.491 & 0.489 & 0.797 & 0.818 \\
\hline $\mathrm{COM}$ & 0.774 & 0.784 & 0.529 & 0.552 \\
\hline$\sim \mathrm{COM}$ & 0.557 & 0.534 & 0.793 & 0.784 \\
\hline PR & 0.598 & 0.757 & 0.469 & 0.613 \\
\hline$\sim \mathrm{PR}$ & 0.694 & 0.559 & 0.814 & 0.676 \\
\hline \multicolumn{5}{|c|}{$\begin{array}{l}\text { Legend: } \sim=\text { negation/absence of condition, Cons }= \\
\text { consistency, } \mathrm{Cov}=\text { coverage, } \mathrm{BI}=\text { behavioral intention, } \\
\mathrm{BE}=\text { benevolence, } \mathrm{COM}=\text { competence, } \mathrm{INT}=\text { integrity, } \\
\mathrm{TAI}=\text { trust in } \mathrm{AI}, \mathrm{DIS}=\text { disposition to trust, } \mathrm{EE}=\text { effort } \\
\text { expectancy, } \mathrm{PE}=\text { performance expectancy, } \\
\mathrm{PR}=\text { perceived privacy risk }\end{array}$} \\
\hline
\end{tabular}

A consistency value above 0.9 implies a necessary condition for an outcome to occur [21]. In our analysis the outcome constitutes the presence of BI or the absence of BI $(\sim \mathrm{BI})$. We could not find any necessary conditions. However, in particular PE for BI and the absence of PR $(\sim \mathrm{PR})$ for $\sim \mathrm{BI}$ as an outcome largely explain the emergence of the respective outcome with high consistency estimates over a large proportion of cases. Nevertheless, the existence of necessary conditions is not inevitably the reason for the intention to use the CA. Thus, we check our data for sufficient conditions.

The results from the analysis of the sufficient conditions are shown in Table 3 . We adopted the notation for presenting the results according to Ragin and Fiss [33]. Black circles “ $\bullet$ " represent the presence and crossed-out circles " $\otimes$ " the absence of conditions. Conditions without a circle describe a "don't care" condition, which characterizes those conditions whose presence or absence plays a subordinate role [21]. While big circles represent core conditions, small symbols iconize peripheral conditions [33].

Table 3. Analysis of sufficient conditions

\begin{tabular}{|c|c|c|c|c|c|c|}
\hline & \multicolumn{2}{|c|}{$\begin{array}{c}\text { BI } \\
\text { configurations }\end{array}$} & \multicolumn{4}{|c|}{$\sim$ BI configurations } \\
\hline & $\mathbf{a}$ & $\mathbf{b}$ & $\mathbf{a}$ & $\mathbf{b}$ & c & d \\
\hline \multicolumn{7}{|c|}{ Purpose dimension } \\
\hline $\mathrm{BE}$ & $\bullet$ & $\bullet$ & $\otimes$ & $\otimes$ & & $\bullet$ \\
\hline TAI & $\bullet$ & & $\otimes$ & $\bullet$ & $\otimes$ & $\otimes$ \\
\hline DIS & & $\bullet$ & & $\otimes$ & $\bullet$ & $\otimes$ \\
\hline \multicolumn{7}{|c|}{ Process dimension } \\
\hline $\mathrm{EE}$ & $\bullet$ & $\bullet$ & & $\otimes$ & $\otimes$ & $\bullet$ \\
\hline INT & $\bullet$ & $\bullet$ & $\otimes$ & $\otimes$ & $\otimes$ & \\
\hline \multicolumn{7}{|c|}{ Performance dimension } \\
\hline PE & ○ & O & $\otimes$ & $\otimes$ & $\otimes$ & $\otimes$ \\
\hline $\mathrm{COM}$ & $\bullet$ & $\bullet$ & $\otimes$ & $\otimes$ & $\otimes$ & $\otimes$ \\
\hline PR & & $\otimes$ & $\otimes$ & & $\otimes$ & $\otimes$ \\
\hline Cons & 0.91 & 0.91 & 0.93 & 0.95 & 0.96 & 0.92 \\
\hline $\mathrm{RCov}$ & 0.43 & 0.32 & 0.43 & 0.3 & 0.33 & 0.29 \\
\hline UCov & 0.13 & 0.03 & 0.07 & 0.06 & 0.02 & 0.05 \\
\hline SCons & \multicolumn{2}{|c|}{0.90} & \multicolumn{4}{|c|}{0.91} \\
\hline SCov & \multicolumn{2}{|c|}{0.45} & \multicolumn{4}{|c|}{0.56} \\
\hline \multicolumn{7}{|c|}{$\begin{array}{l}\text { Legend: } \sim=\text { absence of a condition, } \bullet=\text { presence of a } \\
\text { condition, } \otimes=\text { negation of a condition, big circle }=\text { core } \\
\text { element, small circle = peripheral element, blank space }= \\
\text { subordinate condition, } \\
\mathrm{BI}=\text { behavioral intention, } \mathrm{BE}=\text { benevolence, } \\
\mathrm{COM}=\text { competence, } \mathrm{INT}=\text { integrity, TAI = trust in AI, } \\
\mathrm{DIS}=\text { disposition to trust, } \mathrm{EE}=\text { effort expectancy, } \\
\mathrm{PE}=\text { performance expectancy, } \mathrm{PR}=\text { perceived privacy } \\
\text { risk } \\
\text { Cons = consistency, Cov = coverage, } \mathrm{RCov}=\text { raw } \\
\text { coverage, UCov = unique coverage, SCons = overall } \\
\text { solution consistency, SCov }=\text { overall solution coverage }\end{array}$} \\
\hline
\end{tabular}

The overview in Table 3 comprises two configurational solutions with an overall consistency of 0.903 for BI and four solutions with a value of 0.914 for $\sim \mathrm{BI}$ encompassing core and peripheral conditions. The 
outcome-related total solution coverage is about $50 \%$, which represents a considerable number of empirical cases. We have derived two solutions representing profiles with high CA adoption intentions (BIa, BIb) and four solutions ( $\sim \mathrm{BIa}, \sim \mathrm{BIb}, \sim \mathrm{BIc}, \sim \mathrm{BId})$ that have low adoption intention. The derived outcome-related solutions constitute neutral permutations within solutions demonstrating within-type equifinality [20]. This means that the solutions for $\mathrm{BI}$ and $\sim \mathrm{BI}$ are grouped around a core condition which is manifested by the presence of $\mathrm{PE}$ for $\mathrm{BI}$ and the absence of COM for $\sim \mathrm{BI}$ as an outcome. Furthermore, our identified solutions contain peripheral sufficient conditions. Peripheral conditions represent easy counterfactuals contained in an intermediate solution as an output of the utilized Quine-McCluskey algorithm [21]. Core conditions, by contrast, constitute easy and difficult counterfactuals of a parsimonious solution indicating a stronger causal link to an outcome [63].

\section{Main findings and propositions}

We summarize our main results in light of the employed fsQCA approach, which allows us to exploit the properties of asymmetry, conjunction, and equifinality to interpret our results [35].

Configurations for enhancing the adoption of CAs for disease diagnosis. We identified the configurational solutions BIa and BIb as a conjunctural mix of factors from the purpose, process and performance dimensions that promote the adoption of CAs for disease diagnosis. Even though our results revealed several sufficient conditions for the outcome to occur, the causal link of PE within the acceptance recipes showed the strongest effect. PE constitutes a sufficient core condition and thus is particularly relevant for explaining BI. This is in line with the results of other studies that investigated the importance of performance aspects of AI-based diagnostic systems, as the risk of serious medical consequences when using faulty and inferior systems is perceived as high, particularly in the healthcare sector $[4,5]$. In addition, both configurations are accompanied by peripheral sufficient conditions. The two configurations have certain similarities with the presence of BE, COM, INT and EE and differences in the remaining elements TAI, DIS, PR. These differences manifest themselves in the fact that in $\mathrm{BIb}$ the presence of DIS is associated with the absence of PR, while in BIa the adoption is peripherally influenced by the trust in AI. Therefore, the trust of BIa profiles originate from a good faith whereas BIb profiles rather seek the trust in structural certainty. This could be related to the fact, that many people are unaware of the possible invasion of their privacy or they trust technology despite the potential privacy risk [48].
Proposition 1: The adoption of BI is not explained by single trust-enhancing factors but rather by configurations of several factors from the purpose, process and performance dimensions. PE is a sufficient core condition for the intention to adopt CAs for disease diagnosis. Users who intend to adopt such CAs expect, in particular, to obtain an increase in efficiency in the form of simple and fast diagnostics.

Configurations explaining low adoption intentions toward CAs for disease diagnosis. The complexity and sensitivity of medical diagnostics render it difficult to build trust, even in the diagnoses of doctors [37]. For the profiles $\sim$ BIa, $\sim$ BIb, $\sim$ BIc, $\sim$ BId, a transfer of competence-based trust in the provider of diagnostic applications is not conceivable, especially in view of the high performance expectations [9]. This is evident by the absence of COM as a sufficient core condition in all these configurations. Previous work demonstrated that competence-based trust is mediating the $\mathrm{PE}$ of received knowledge [64]. Our results confirm this in context of CAs and show that it has the strongest causal effect to explain intentions not to adopt the technology. Therefore, the performance dimension is also crucial for low adoption intentions, but in this case the institutional level is addressed, i.e., the service provider, as opposed to high adoption intentions, where the essential competence must be demonstrated at the artefact level. However, as with the causality for the acceptance of CAs, low adoption intentions are caused by a conjunction of various absent and present conditions of factors that explain $\sim$ BI. Yet these other factors have a peripheral influence on the outcome.

Proposition 2: There are more profiles that explain a low level of adoption intentions of CAs in disease diagnosis than a high level. All these configurations are strongly and sufficiently influenced by the absence of COM and represent neutral permutations around this core condition.

\section{Discussion}

There is a broad consensus in IS literature on the significance of trust for the intention to adopt CAs in healthcare $[5,10]$. Our configurational approach reinforces this notion and emphasizes the conditions of trust-enhancing factors affecting the behavioral intention to adopt CAs for disease diagnosis. Driven by the COVID-19 pandemic in 2020, the need for indirect medical advice increased significantly. As our results enhance the understanding of users' motives to adopt CAs and increase transparency of the inherent process of establishing initial trust in AI in healthcare, they are valuable for both research and practice. First, we showed that trust-building towards AI-based artefacts is a complex process that can follow different causal 
pathways. Specifically, we demonstrated that a mix of trust-enhancing factors related to the dimensions purpose, performance and process can form a composite of different effects explaining BI and $\sim \mathrm{BI}$. This supports Lee and See [15] and Hengstler et al. [13], whose research emphasized these dimensions in the formation of trust in the context of automation and applied AI. We also revealed that the adoption of CAs, particularly in the initial stage, cannot be explained by a specific set of universal factors, but rather by configurations of factors that correspond to certain trust profiles indicating within-type equifinality. In doing so, we followed the call for further research, especially in the investigation of different conditions for the adoption of AI-based digital assistants [5, 65]. In addition, we demonstrated the usefulness of configurational approaches for such research questions. Aside from these implications, our study focuses on users testing Ada for the first time, so we consider the initial stage of the dynamic formation of trust. We contribute to the discussion on trust in AI $[4,30]$ by identifying specific performance aspects that are especially important in the initial phase of adoption. In addition, practitioners benefit from our findings. PE was identified as a core condition for the adoption of CAs. Although apps like Ada are already technologically sophisticated, further development is needed to make the technology more performant and reliable. Yet, this mainly depends on data availability and demands that policy makers create appropriate conditions allowing a safe use of medical data [26]. Furthermore, Meier et al. [6] emphasized the importance of the usability of CAs in context of requirements engineering. In line with this research, we found that effort expectancy together with institutionbased trust constitutes peripheral conditions for the BI to use the CA. Thus, a CA for medical diagnosis should be easy to use for potential user groups, e.g., by enabling a goal-oriented conversational flow and a domainspecific understanding as well as creating a user-centred design and an information-focused user interface. Our configurations contribute to adapting the personality of a CA to the expectations of users, as addressed by Meier et al. [6] as an important requirement in CA engineering. To prevent rejection, providers of CAs should emphasize their competence in developing such technologies and possessing diagnostic expertise.

\section{Conclusion, limitations and directions for future research}

As every research, this study is subject to some limitations. Since our online survey was conducted among students of a German university, the findings are limited and do not include the perceptions and attitudes of other groups such as adult patients and elderly. Thus, our study sample may be biased with regard to age, education and digital literacy as well as geographic and cultural influences towards specific user types. Further research is necessary to extend the scope of this study to a broader data base and thus allow for a more comprehensive picture of trust configurations affecting the BI to adopt CAs. Another limitation concerns the research model derived from prior IS acceptance research. Despite the thorough literature review, we cannot guarantee that we have captured all relevant constructs and contributions. Furthermore, given the novelty of the topic, prior studies might be insufficient as a single data base for our research model. Instead, it might be worthwhile to complement the knowledge base by insights gained from primary data (e.g. qualitative interviews) to achieve a more comprehensive understanding of the topic. The third limitation refers to the employed analysis method. As emphasized by Ragin and Fiss [33], the explanatory power of fsQCA can be enhanced by complementary quantitative or qualitative methods. Thus, the results of our study should be complemented by and compared with findings based on the application of other statistical analyses such as structural equation modeling or qualitative case studies. Another limitation of this study is concerned with the absence of moderator variables in our research model. IS scholars have emphasized the need to include user conditions as an important antecedent of the intention to adopt CAs into the scope of future studies [5]. Future research should focus on moderating effects such as gender or the health status to provide a more granular and differentiated view on the BI to adopt CAs for medical diagnosis.

Despite the indisputable potential of CAs in healthcare, their dissemination is still low [66]. We identified trust-enhancing factors that promote the understanding of the adoption process of CAs for disease diagnosis. Our configurational approach provides a detailed picture of users' intentions by explaining how trust-enhancing factors related to the purpose, process and performance dimensions interact to yield the same outcome. Based on our findings, future research, marketing strategies and product designs could be adopted accordingly, thus promoting the dissemination of CAs for medical diagnosis.

\section{References}

[1] Kearley, K.E., G.K. Freeman, and A. Heath, "An exploration of the value of the personal doctor-patient relationship in general practice.", The British journal of general practice: the journal of the Royal College of General Practitioners 51(470), 2001, pp. 712-8.

[2] Rochaix, L., "Information asymmetry and search in the market for physicians' services", Journal of Health Economics 8(1), 1989, pp. 53-84. 
[3] Skirbekk, H., A.-L. Middelthon, P. Hjortdahl, and A. Finset, "Mandates of Trust in the Doctor-Patient Relationship", Qualitative Health Research 21(9), 2011, pp. 1182-1190.

[4] Fan, W., J. Liu, S. Zhu, and P.M. Pardalos, "Investigating the impacting factors for the healthcare professionals to adopt artificial intelligence-based medical diagnosis support system (AIMDSS)", Annals of Operations Research, 2018, pp. 1-26.

[5] Laumer, S., C. Maier, and F.T. Gubler, "CHATBOT ACCEPTANCE IN HEALTHCARE: EXPLAINING USER ADOPTION OF CONVERSATIONAL AGENTS FOR DISEASE DIAGNOSIS", European Conference on Information Systems, (2019).

[6] Meier, P., J. Beinke, C. Fitte, A. Behne, and F. Teuteberg, "FeelFit - Design and Evaluation of a Conversational Agent to Enhance Health Awareness", International Conference on Information Systems, 2019.

[7] Raghupathi, W., and V. Raghupathi, "Big data analytics in healthcare: promise and potential", Health Information Science and Systems 2(1), 2014, pp. 3.

[8] Ada Health Limited, “Ada Health”, 2020. https://ada.com/

[9] Lai, Y., E. Lioliou, and P. Panagiotopoulos, "UNDERSTANDING USERS' SWITCHING INTENTION TO AI-POWERED HEALTHCARE CHATBOTS", European Conference on Information Systems, (2021).

[10] Prakash, A.V., and S. Das, "Would you Trust a Bot for Healthcare Advice? An Empirical Investigation", Pacific Asia Conference on Information Systems, (2020).

[11] Mesbah, N., and L. Pumplun, "'HELLO, I'M HERE TO HELP YOU' - MEDICAL CARE WHERE IT IS NEEDED MOST: SENIORS' ACCEPTANCE OF HEALTH CHATBOTS", European Conference on Information Systems, (2020).

[12] Seitz, L., J. Woronkow, S. Bekmeier-Feuerhahn, and K. Gohil, "THE ADVANCE OF DIAGNOSIS CHATBOTS: SHOULD WE FIRST AVOID DISTRUST BEFORE WE FOCUS ON TRUST?", European Conference on Information Systems, (2021).

[13] Hengstler, M., E. Enkel, and S. Duelli, “Applied artificial intelligence and trust-The case of autonomous vehicles and medical assistance devices", Technological Forecasting and Social Change 105, 2016, pp. 105-120.

[14] Ye, T., J. Xue, M. He, et al., "Psychosocial Factors Affecting Artificial Intelligence Adoption in Health Care in China: Cross-Sectional Study", Journal of medical Internet research 21(10), 2019, pp. e14316.

[15] Lee, J.D., and K.A. See, "Trust in Automation: Designing for Appropriate Reliance", Human Factors: The Journal of the Human Factors and Ergonomics Society 46(1), 2004, pp. 50-80.

[16] Söllner, M., A. Hoffmann, and J.M. Leimeister, "Why different trust relationships matter for information systems users", European Journal of Information Systems 25(3), 2016, pp. 274-287.

[17] Benbasat, I., and W. Wang, "Trust In and Adoption of Online Recommendation Agents", Journal of the Association for Information Systems 6(3), 2005, pp. 72 101.

[18] Walterbusch, M., M. Gräuler, and F. Teuteberg, "How trust is defined: A qualitative and quantitative analysis of scientific literature", 20th Americas Conference on Information Systems, 2014.

[19] Vis, B., "The Comparative Advantages of fsQCA and Regression Analysis for Moderately Large-N Analyses", Sociological Methods \& Research 41(1), 2012, pp. 168198.

[20] Fiss, P.C., "Building Better Causal Theories: A Fuzzy Set Approach to Typologies in Organization Research", Academy of Management Journal 54(2), 2011, pp. 393420.

[21] Leonhardt, D., P. Huang, A. Hanelt, and S. Mithas, "Does one size fit all? Theorizing governance configurations for digital innovation", International Conference on Information Systems 2018, (2018).

[22] Diederich, S., A.B. Brendel, and L.M. Kolbe, "Towards a Taxonomy of Platforms for Conversational Agent Design", 14th International Conference on Wirtschaftsinformatik, (2019).

[23] Palanica, A., P. Flaschner, A. Thommandram, M. Li, and Y. Fossat, "Physicians' Perceptions of Chatbots in Health Care: Cross-Sectional Web-Based Survey", Journal of Medical Internet Research 21(4), 2019, pp. e12887.

[24] PWC, "How artificial intelligence may improve quality and efficiency, whilst reducing healthcare costs in Europe", Sherlock in Health: How artificial intelligence may improve quality and efficiency, whilst reducing healthcare costs in Europe(June), 2017, pp. 24.

[25] European Commission, "High-Level Expert Group on Artificial Intelligence", 2019, pp. 2-36.

[26] Sun, T.Q., and R. Medaglia, "Mapping the challenges of Artificial Intelligence in the public sector: Evidence from public healthcare", Government Information Quarterly 36(2), 2019, pp. 368-383.

[27] Diederich, S., A.B. Brendel, S. Morana, and L. Kolbe, "On the Design of and Interaction with Conversational Agents: An Organizing and Assessing Review of Human-Computer Interaction Research", Journal of the Association for Information Systems forthcomin, 2022.

[28] Gefen, D., "E-commerce: the role of familiarity and trust", Omega 28(6), 2000, pp. 725-737.

[29] McKnight, H., M. Carter, and P. Clay, "Trust in Technology: Development of a Set of Constructs and Measures", DIGIT 2009 Proceedings - Diffusion Interest Group in Information Technology, 2009, pp. 12.

[30] Siau, K., and W. Wang, "Building trust in artificial intelligence, machine learning, and robotics", Cutter business technology journal 31(2), 2018, pp. 47-53.

[31] McKnight, D.H., V. Choudhury, and C. Kacmar, "Developing and Validating Trust Measures for eCommerce: An Integrative Typology", Information Systems Research 13(3), 2002, pp. 334-359.

[32] Gefen, D., E. Karahanna, and D.W. Straub, "Inexperience and experience with online stores: The importance of tam and trust", IEEE Transactions on Engineering Management 50(3), 2003, pp. 307-321.

[33] Ragin, C.C., and P.C. Fiss, "Net effects analysis versus configurational analysis: An empirical demonstration", In C.C. Ragin, ed., Redesigning social inquiry: Fuzzy sets and beyond. University of Chicago Press, Chicago, 2008, 190-212. 
[34] Wang, W., and K. Siau, "Trust in Health Chatbots", International Conference on Information Systems, (2018).

[35] Park, Y., P.C. Fiss, and O.A. El Sawy, "Theorizing the Multiplicity of Digital Phenomena: The Ecology of Configurations, Causal Recipes, and Guidelines for Applying QCA", MIS Quarterly 44(4), 2020, pp. 14931520.

[36] Lee, J., and N. Moray, "Trust, control strategies and allocation of function in human-machine systems", Ergonomics 35(10), 1992, pp. 1243-1270.

[37] Calnan, M., and R. Rowe, "Trust and Health Care", Sociology Compass 1(1), 2007, pp. 283-308.

[38] LaRosa, E., and D. Danks, "Impacts on Trust of Healthcare AI", Proceedings of the 2018 AAAI/ACM Conference on AI, Ethics, and Society, ACM (2018), 210-215.

[39] DeCamp, M., and J.C. Tilburt, "Why we cannot trust artificial intelligence in medicine", The Lancet Digital Health 1(8), 2019, pp. e390.

[40] McKnight, D.H., V. Choudhury, and C. Kacmar, "Developing and Validating Trust Measures for eCommerce: An Integrative Typology", Information Systems Research 13(3), 2002, pp. 334-359.

[41] Venkatesh, V., J.Y.L. Thong, and X. Xu, "Consumer acceptance and use of information technology: extending the unified theory of acceptance and use of technology", MIS Quarterly 36(1), 2012, pp. 157-178.

[42] Venkatesh, Morris, Davis, and Davis, "User Acceptance of Information Technology: Toward a Unified View", MIS Quarterly 27(3), 2003, pp. 425.

[43] Xu, N., and K.-J. Wang, "Adopting robot lawyer? The extending artificial intelligence robot lawyer technology acceptance model for legal industry by an exploratory study", Journal of Management \& Organization(2019), 2019, pp. 1-19.

[44] Davis, F.D., "Perceived Usefulness, Perceived Ease of Use, and User Acceptance of Information Technology", MIS Quarterly 13(3), 1989, pp. 319.

[45] Venkatesh, V., and F.D. Davis, "A Theoretical Extension of the Technology Acceptance Model: Four Longitudinal Field Studies", Management Science 46(2), 2000, pp. 186-204.

[46] Mayer, R.C., J.H. Davis, and F.D. Schoorman, "An Integrative Model Of Organizational Trust", Academy of Management Review 20(3), 1995, pp. 709-734.

[47] Pavlou, P.A., "Consumer Acceptance of Electronic Commerce: Integrating Trust and Risk with the Technology Acceptance Model", International Journal of Electronic Commerce 7(3), 2003, pp. 101-134.

[48] Nienaber, A., and G. Schewe, "ENHANCING TRUST OR REDUCING PERCEIVED RISK, WHAT MATTERS MORE WHEN LAUNCHING A NEW PRODUCT?", International Journal of Innovation Management 18(01), 2014, pp. 1450005.

[49] Fiss, P.C., "A set-theoretic approach to organizational configurations", Academy of Management Review 32(4), 2007, pp. 1180-1198.

[50] Lee, J.-N., Y. Park, D.W. Straub, and Y. Koo, "Holistic archetypes of IT outsourcing strategy: A contingency fit and configurational approach", MIS Quarterly:
Management Information Systems 43(4), 2019, pp. 12011225.

[51] Pappas, I.O., and A.G. Woodside, "Fuzzy-set Qualitative Comparative Analysis (fsQCA): Guidelines for research practice in Information Systems and marketing", International Journal of Information Management 58, 2021, pp. 102310.

[52] Compeau, D., B. Marcolin, H. Kelley, and C. Higgins, "Research Commentary - Generalizability of Information Systems Research Using Student SubjectsA Reflection on Our Practices and Recommendations for Future Research", Information Systems Research 23(4), 2012, pp. 1093-1109.

[53] Moser, C.A., Survey Methods in Social Investigation, Routledge, 2017.

[54] Nunnally, J.C., and I.H. Bernstein, Psychometric theory, McGraw-Hill, New York, 1994.

[55] Podsakoff, P.M., S.B. MacKenzie, J.-Y. Lee, and N.P. Podsakoff, "Common method biases in behavioral research: A critical review of the literature and recommended remedies.", Journal of Applied Psychology 88(5), 2003, pp. 879-903.

[56] Fornell, C., and D.F. Larcker, "Structural Equation Models with Unobservable Variables and Measurement Error: Algebra and Statistics", Journal of Marketing Research 18(3), 1981, pp. 382-388.

[57] Henseler, J., C.M. Ringle, and M. Sarstedt, "A new criterion for assessing discriminant validity in variancebased structural equation modeling", Journal of the Academy of Marketing Science 43(1), 2015, pp. 115-135.

[58] Schneider, C.Q., and C. Wagemann, Set-Theoretic Methods for the Social Sciences, Cambridge University Press, Cambridge, 2012.

[59] Duarte, R., and W. Picoto, "APPLYING CONFIGURATIONAL THEORY TO UNDERSTAND MOBILE APP SUCCESS", ECIS, (2016).

[60] Leischnig, A., S. Woelfl, and B.S. Ivens, "When Does Digital Business Strategy Matter to Market Performance?", ICIS, (2016).

[61] Ragin, C.C., "Set Relations in Social Research: Evaluating Their Consistency and Coverage", Political Analysis 14(3), 2006, pp. 291-310.

[62] Park, Y., and S. Mithas, "Organized Complexity of Digital Business Strategy: A Configurational Perspective", MIS Quarterly 44(1), 2020, pp. 85-127.

[63] Misangyi, V.F., and A.G. Acharya, "Substitutes or Complements? A Configurational Examination of Corporate Governance Mechanisms", Academy of Management Journal 57(6), 2014, pp. 1681-1705.

[64] Levin, D.Z., and R. Cross, "The strength of weak ties you can trust: The mediating role of trust in effective knowledge transfer", Management Science 50(11), 2004, pp. 1477-1490.

[65] Maedche, A., C. Legner, A. Benlian, et al., "AI-Based Digital Assistants: Opportunities, Threats, and Research Perspectives", Business and Information Systems Engineering, 2019.

[66] Laranjo, L., A.G. Dunn, H.L. Tong, et al., "Conversational agents in healthcare: a systematic review", Journal of the American Medical Informatics Association 25(9), 2018, pp. 1248-1258. 